\title{
MicroRNA-125b regulates the expression of aggrecanase-1 (ADAMTS-4) in human osteoarthritic chondrocytes
}

\author{
Tetsuya Matsukawa ${ }^{1,2}$, Tadahiro Sakai ${ }^{1 *}$, Tomo Yonezawa ${ }^{2,3}$, Hideki Hiraiwa ${ }^{1}$, Takashi Hamada ${ }^{1}$, \\ Motoshige Nakashima', Yohei Ono', Shinya Ishizuka', Hiroyuki Nakahara², Martin K Lotz ${ }^{2}$, Hiroshi Asahara ${ }^{2,4}$ and \\ Naoki Ishiguro' ${ }^{1}$
}

\begin{abstract}
Introduction: Increased expression of aggrecanase-1 (ADAMTS-4) has emerged as an important factor in osteoarthritis $(\mathrm{OA})$ and other joint diseases. This study aimed to determine whether the expression of ADAMTS-4 in human chondrocytes is regulated by miRNA.

Methods: MiRNA targets were identified using bioinformatics. Chondrocytes were isolated from knee cartilage and treated with interleukin-1 beta (IL-1 $\beta$ ). Gene expression was quantified using TaqMan assays and protein production was determined by immunoblotting. Luciferase reporter assay was used to verify interaction between miRNA and target messenger RNA (mRNA).

Results: In silico analysis predicted putative target sequence of miR-125b on ADAMTS-4. MiR-125b was expressed in both normal and OA chondrocytes, with significantly lower expression in OA chondrocytes than in normal chondrocytes. Furthermore, IL-1 $\beta$-induced upregulation of ADAMTS- 4 was suppressed by overexpression of miR$125 \mathrm{~b}$ in human OA chondrocytes. In the luciferase reporter assay, mutation of the putative miR-125b binding site in the ADAMTS-4 3'UTR abrogated the suppressive effect of miR125.
\end{abstract}

Conclusions: Our results indicate that miR-125b plays an important role in regulating the expression of ADAMTS-4 in human chondrocytes and this identifies miR-125b as a novel therapeutic target in OA.

\section{Introduction}

Osteoarthritis (OA) is the most common form of arthritis and is characterized by loss of articular cartilage, which is ascribed mainly to proteolysis of the structural components of the extracellular matrix (ECM), that is, proteoglycans and collagens. Collagen degradation is principally carried out by several proteinases shedding an ectodomain of membrane proteins belonging to the matrix metalloproteinase (MMP) family, such as MMP1, MMP8, and MMP13 [1-3]. On the other hand, aggrecanases represent another class of proteinases belonging to the ADAMTS (a disintegrin and metalloproteinase with thrombospondin motifs) family. Biochemical

\footnotetext{
* Correspondence: tadsakai@med.nagoya-u.ac.jp

'Department of Orthopaedic Surgery, Nagoya University Graduate School of Medicine, 65 Tsurumai-cho, Showa-ku, Nagoya 466-8550, Japan

Full list of author information is available at the end of the article
}

studies indicate that ADAMTS $1,4,5,8,9$, and 15 possess aggrecanase activity [4,5]. Song et al. [6] reported that knockdown of aggrecanase-1 (ADAMTS-4), aggrecanase-2 (ADAMTS-5), or both enzymes by small interfering RNA attenuates the degradation of aggrecan in human cartilage stimulated by a combination of both tumor necrosis factor-alpha (TNF- $\alpha$ ) and oncostatin M. This suggests that both ADAMTS- 4 and ADAMTS- 5 contribute to aggrecanolysis in human tissue. Recent studies showed that ADAMTS-4 is selectively overexpressed in human OA cartilage, with a positive correlation with the degree of cartilage destruction, whereas ADAMTS- 5 is similarly expressed in both normal and OA cartilages [7]. These results suggest that ADAMTS4 is a major aggrecanase in human OA cartilage and its induction is involved in the pathogenesis of OA.

\section{Biomed Central}


Proinflammatory cytokines secreted from chondrocytes such as interleukin-1 beta (IL-1 $\beta$ ) and TNF- $\alpha$ contribute to the progression of OA [8-10]. IL-1 $\beta$ induces a cascade of inflammatory and catabolic events in chondrocytes. It also changes chondrocyte anabolism by suppressing the synthesis of proteoglycans and collagens [9,11-13].

MicroRNAs (miRNAs), which belong to a class of short 19 to 23 nucleotide non-coding RNAs, function as posttranscriptional negative regulators by promoting messenger RNA (mRNA) degradation or repressing translation through complementarily binding target sequences in the 3'-untranslated regions (3'-UTRs) of specific mRNA targets [14-17]. Degradation of target mRNAs depends on near-perfect complementarity while for repression of translation partial complementarity is sufficient [18].

Recent studies have identified the miRNA expression profiles in human OA chondrocytes $[19,20]$, but no consensus on a specific miRNA being the regulator of ADAMTS-4 expression has been reached. In this study, we use bioinformatics to predict putative target sequences for miR-125a, miR-125b and miR-4319 in human ADAMTS-4 mRNA. We demonstrate that expression of miR-125b, but not miR-125a and miR4319 , is significantly decreased in human OA chondrocytes compared to normal chondrocytes. This prompted us to validate whether ADAMTS-4 is regulated by miR$125 \mathrm{~b}$ in human OA chondrocytes. We also established a reporter system using the 3'-UTR of human ADAMTS4 mRNA, and showed that this is targeted by miR-125b.

\section{Materials and methods Specimen selection}

Cartilage tissues were aseptically obtained at the time of total knee replacement from OA patients diagnosed according to the American College of Rheumatology criteria for this disease. The patients with Kellgren-Lawrence OA grades II, III, and IV included five females and two males, with a mean age of 74.1 years (ranging from 68 to 82 years). Articular cartilage from the weight-bearing, fibrillated areas of the femoral condyle and tibial plateau was cut into small pieces with a scalpel. Cartilage samples were minced and digested at $37^{\circ} \mathrm{C}$ with trypsin-EDTA solution for $30 \mathrm{~min}$, incubated with 3 $\mathrm{mg} / \mathrm{ml}$ type II collagenase (Worthington Biochemical Co, Lakewood, NJ, USA) in Dulbecco's modified Eagle's medium (DMEM; Gibco-BRL, Life Technologies, Merelbeke, Belgium) for $18 \mathrm{~h}$, filtered through a nylon mesh, and washed extensively. Next, the isolated chondrocytes were seeded in $75 \mathrm{~cm}^{2}$ culture flasks and incubated in DMEM containing $10 \%$ fetal bovine serum (FBS), penicillin $(100 \mathrm{U} / \mathrm{ml})$, and streptomycin $(100 \mathrm{mg} / \mathrm{ml})$ at $37^{\circ} \mathrm{C}$ in an atmosphere of $5 \%$ CO2. At confluence, the cells were detached and seeded in 12-well plates at a density of $2 \times 10^{5} /$ well. These experiments were performed in accordance with a protocol approved by the Ethics Committee of Nagoya University and all patients gave written consent for the use of their tissues for this research. Normal human articular chondrocytes from knee joints including three males, with a mean age of 37.6 years (ranging from 34 to 45 ) were purchased from Takara Bio Inc. (Shiga, Japan). Cartilage tissues for the direct isolation of microRNA, were harvested from human knee joints including three male, with a mean age of 18.3 years (ranging from 17 to 19) for normal samples (OA grade I), one male and two females, with a mean age of 57.7 years (ranging from 54 to 65) for OA samples (OA grade IV) and two male and one female, with a mean age of 50.7 years (ranging from 47 to 55) for normal age-matched (aging) samples (OA grade I), which were obtained from the tissue banks with approval of the Scripps Human Subjects Committee. Joints were processed within 72 hours postmortem.

\section{Cell culture}

Articular cartilage tissues were cut into small pieces less than $1 \mathrm{~mm}^{3}$, and subsequently digested with $1 \mathrm{mg} / \mathrm{mL}$ collagenase from Clostridium histolyticum (SigmaAldrich Co., Tokyo, Japan) at $37^{\circ} \mathrm{C}$ for $16 \mathrm{~h}$ with shaking. Isolated primary cultured human OA articular chondrocytes and 293T cells were cultured in DMEM containing $10 \% \mathrm{FBS}$ and $1 \%$ penicillin/streptomycin (Gibco-BRL, Life Technologies, Grand Island, NY, USA) at $37^{\circ} \mathrm{C}$ in a humidified atmosphere of $5 \% \mathrm{CO}_{2}$. The medium was changed every three days.

\section{Treatment with IL-1 $\beta$ and preparation of miRNA}

Primary cultured human chondrocytes were serumstarved overnight and then treated with recombinant human IL-1 $\beta$ (5 ng/mL; PeproTech, Rocky Hill, NJ, USA) for the indicated periods of time. Total RNA containing miRNA was extracted using the mirVana miRNA isolation kit (Applied Biosystems, Life Technologies, Carlsbad, CA, USA). Cells were collected by Lysis/Binding Buffer including 1/10 volume of miRNA Homogenate Additive and then Acid-Phenol:Chloroform was added. After centrifugation, the aqueous phase was transferred to fresh tubes and, after addition of 1.25 volumes of $100 \%$ ethanol, applied to a filter cartridge. After washing the filter, total RNA was extracted with nuclease-free water. Total RNA of cartilage tissue was isolated from fresh-frozen cartilage by homogenizing the tissue in a freezer mill (Spex, Metuchen, NJ, USA) and extracting the homogenate in TRIzol (Invitrogen, Life Technologies, Carlsbad, CA, USA). 


\section{Transfection}

MiRNA precursor (pre-miR-125b), antagomir (anti-miR125b), or negative control oligonucleotides were obtained from Applied Biosystems. Primary cultured human chondrocytes were transfected with each oligonucleotides using Lipofectamine 2000 (Invitrogen, Life Technologies). Forty-eight hours after transfection, the cells were serum-starved overnight and then cultured in serum-free DMEM in the presence or absence of recombinant human IL-1 $\beta$ (5 ng/mL; PeproTech) for 24 hours. Cells were harvested and subjected to total RNA and protein extraction. HEK $293 \mathrm{~T}$ cells were transfected with $1 \mu \mathrm{g}$ reporter plasmid, $1 \mu \mathrm{g}$ effector plasmid or point mutant of pre-miR-125b plasmid or negative control plasmid, and $10 \mathrm{ng}$ of Renilla luciferase control vector using FuGENE HD Transfection Reagent (Promega, Madison, WI, USA) in 24-well plates. The detail of all constructs is described in 'Luciferase reporter assay'.

\section{Reverse transcription}

Total RNA concentration was determined using absorption spectrochemical analysis. Ten nanograms of total RNA containing miRNA from control and experimental samples were reverse-transcribed using miR-125b stemloop RT primer, 10× RT buffer, 100 mM deoxynucleoside triphosphates (dNTPs), 50 units of MultiScribe reverse transcriptase, and 20 units of RNase inhibitor at $16^{\circ} \mathrm{C}$ for $30 \mathrm{~min}, 42^{\circ} \mathrm{C}$ for $30 \mathrm{~min}$, and $85^{\circ} \mathrm{C}$ for $5 \mathrm{~min}$, and then the miRNA product was maintained at $4^{\circ} \mathrm{C}$. Complementary DNA (cDNA) was produced using a High Capacity cDNA Reverse Transcription Kit (Applied Biosystems). Reverse transcription of cDNA from control and experimental samples was performed in $2 \mu \mathrm{L}$ of $10 \times$ Reverse Transcription Buffer, $0.8 \mu \mathrm{L}$ of $25 \times \mathrm{dNTP}$, $2 \mu \mathrm{L}$ of $10 \times$ Random Primers, $1 \mu \mathrm{L}$ of 50 units of MultiScribe Reverse Transcriptase, and $1 \mu \mathrm{L}$ of RNase inhibitor at $25^{\circ} \mathrm{C}$ for $10 \mathrm{~min}, 37^{\circ} \mathrm{C}$ for $120 \mathrm{~min}$, and $85^{\circ} \mathrm{C}$ for $5 \mathrm{~s}$, and then the cDNA product was maintained at $4^{\circ} \mathrm{C}$.

\section{Quantitative RT-PCR analysis of ADAMTS-4, ADAMTS-5, and miR-125b}

Expression of ADAMTS-4, ADAMTS-5, glyceraldehyde 3-phosphate dehydrogenase (GAPDH) mRNA, mature miR-125a, miR-125b, miR-4319 or U6 small nuclear RNA (RNU6B) was determined using the TaqMan Gene Expression Assay (Applied Biosystems). The reactions for miRNA were performed in $20 \mu \mathrm{L}$ of final volume containing $1.33 \mu \mathrm{L}$ of RT product, $10 \mu \mathrm{L}$ of $2 \times$ TaqMan Universal Master Mix, $1 \mu \mathrm{L}$ of $20 \times$ Custom TaqMan Small RNA Assay, and 7.67 $\mu \mathrm{L}$ of nuclease-free water. The reactions for mRNA were performed in $20 \mu \mathrm{L}$ of final volume containing $9 \mu \mathrm{L}$ of RT product, $10 \mu \mathrm{L}$ of $2 \times$ TaqMan Universal Master Mix, and $1 \mu \mathrm{L}$ of $20 \times$ TaqMan Assay. To reduce variability among replicates,
PCR premixes, which contain all reagents except for the template, were prepared and aliquotted into $0.2 \mathrm{ml}$ thin well plates. The samples were incubated at $95^{\circ} \mathrm{C}$ for 10 min, followed by 40 cycles at $95^{\circ} \mathrm{C}$ for $30 \mathrm{~s}$ and $60^{\circ} \mathrm{C}$ for 1 min. Expression of GAPDH and RNU6B were used as internal controls to normalize each mRNA and miRNA expression, respectively. A threshold cycle was observed in the exponential phase of amplification, and quantification of relative expression levels was determined by the $\Delta \Delta C_{t}$ method. The value of each control sample was set at 1 and was used to calculate the fold change in target genes.

\section{Immunoblotting}

Cell lysates were prepared using RIPA buffer (sc-24948; Santa Cruz Biotechnology, Santa Cruz, CA, USA), subjected to sodium dodecyl sulfate-polyacrylamide gel electrophoresis (SDS-PAGE), and then transferred to nitrocellulose membranes (Bio-Rad, Hercules, CA, USA). Membranes were blocked with 5\% nonfat dry milk in Tris-buffered saline containing $0.1 \%$ Tween 20 and then treated with diluted $(1: 1,000)$ polyclonal antibodies specific for ADAMTS-4 (Cosmo Bio Co. Ltd., Tokyo, Japan) or $\beta$ actin (Cell Signaling Technology, Danvers, MA, USA). Immunoreactive proteins were visualized using horseradish peroxidase-conjugated secondary antibodies and enhanced chemiluminescence (Thermo Scientific, Tokyo, Japan). Images were captured using AE-9150 Ez-Capture II (Atto, Tokyo, Japan) and analyzed using CS Analyzer version 3.0 (Atto). Each band was scanned with background correction, and values were averaged and expressed as the mean \pm standard deviation (SD).

\section{Luciferase reporter assay}

The luciferase Vector pLuc2 was used to construct a reporter plasmid containing 1,389 base pairs of the 3'-UTR of human ADAMTS-4 mRNA (uc001fyu.2 obtained from UCSC genome browser [21]) containing two parts of the predicted complementary seed sequence for miR-125b (sequence of mature form: UCCCUGAGACCCUAACUUGUGA). Total RNA (1 $\mu \mathrm{g})$ from human chondrocytes was reverse-transcribed into cDNA, and the ADAMTS-4 3'-UTR was amplified using primers (Forward;AAGATATCGGGGAGAACCCACAGGGAGACC and Reverse;TTGGTACCGAGTGTTATGCTAGTTCTTTATTTACATTATTTAATCCTCAC). PCR products and vectors were digested with EcoRV and Kpn I restriction endonucleases (New England Biolabs, Ipswich, MA, USA), digested vectors were dephosphorylated by calf intestinal phosphatase (CIP) and ligated $3^{\prime}$ to the luciferase reporter gene using the EcoRV and Kpn I site to generate pLuc2-ADAMTS-4 plasmid (reporter plasmid). We found two putative miR- 
$125 \mathrm{~b}$ sequences in the human genome, termed pre-miR125b-1 (encoded on chromosomes 11) (uc010rzr.1) and pre-miR-125b-2 (encoded on chromosomes 21) (uc002ykf.3), respectively. Genomic sequences containing precursors of miR-125b were amplified from DNA from human chondrocytes using primers (miR125b-1 Forward;AAGGATCCCTTAGAGAAGAAATACCATACCACCTGTTTGTTGC and Reverse;TTGATATCACCTCAGACAAACTTTCTTTTCTTTTGTTTTTG CTTTAAAG, miR125b-2 Forward;AAGGATCCTTTCTACTGAAGTATTTTAAATAGTATTTAGAGGTA AAAGTCTAAGTG and Reverse;TTGATATCCTGATGATAAAGAAAAGCATTGTTCTTTTCTCCTAGGC). Each pre-miR cassette was digested with BamH I and EcoRV restriction endonucleases (New England Biolabs). Digested vectors were dephosphorylated by CIP and ligated into the pcDNA3.1 vector using the BamH I and EcoRV site to generate pcDNA3.1-pre-miR-125b-1 or -2 plasmid (effector plasmid). Using site-directed mutagenesis, we inserted several mutations into the seed sequence of miR-125b-1, miR125b-2 and ADAMTS-4 3'-UTR. Mutated vectors of miR-125b precursors were amplified from each effector plasmid template using primers (miR-125b-1 mutation1 Forward;AGAAAACATTGTTGCGCTCCTCTCAGtgGGtgTCACCCTA ACTTGTGATGTTTACCG and Reverse;CGGTAAACATCACAAGTTAGGGTGACACCCACTGAGAGGAGCGCAACAATGTTTTCT, miR-125b-1 mutation2 Forward;ACCGTTTAAATCCACGGGTTAGGCt-

GAtgCCacCTGCGAGTCGTGCTTTTGCATCCT and Reverse;AGGATGCAAAAGCACGACTCGCAGGTGGCATCAGCCTAACCCGTGGATTTAAACGGT, miR125b-2 mutation1 Forward;TCTACCGCATCAAACCAGACTTTTCCTAGtgGGtgTCACCCTAACTTGTGA GGTATTTTAGTAA and Reverse;TTACTAAAATACCTCACAAGTTAGGGTGACACCCACTAGGA AAAGTCTGGTTTGATGCGGTAGA, miR-125b-2 mutation2 Forward;AGGTATTTTAGTAACATCACAAGTCAGGCtGAtgCCacCTAGGCGGAGGGGAACCAGCAGCTTTG and Reverse;CAAAGCTGCTGG TTCCCCTCCGCCTAGGTGGCATCAGCCTGACT TGTGATGTTACTAAAATACCT; the bold letters indicate mutations. the uppercase letters indicate complementary binding in the precursor). Mutated vectors of ADAMTS-4 3'-UTR were amplified from reporter plasmid template using primers (ADAMTS-4 3'-UTR point mutation 1 Forward;GTCAAGGGTAGGGTGGGCCttGaCCCaGTGAGGGATTATCT and Reverse;AGATAATCCCTCACTGGGTCAAGGCCCACCCTACCC TTGAC, ADAMTS-4 3'-UTR point mutation 2 Forward; GAACTCCTGACCAGaCCtaATCGACCTGCCT and Reverse; AGGCAGGTCGATTAGGTCTGGTCAGGAGTTC; the bold letters indicate mutations. the uppercase letters indicate complementary binding in the
ADAMTS-4 3'-UTR). After DpnI digestion, digested vectors were transformed, and then single colonies were picked from ampicillin agar plates, and plasmid DNA was prepared using a Plasmid Miniprep Kit (Qiagen, Valencia, CA, USA). Finally, we confirmed the sequence of established constructs as described above. The luciferase activity assay was performed 24 hours after transfection, using the Dual-Luciferase Reporter Assay System (Promega) and a VICTOR $X$ Light Luminescence Plate Reader (PerkinElmer, Waltham, MA, USA). Firefly luciferase activity was normalized to Renilla luciferase activity. Each experiment was performed three times in triplicate

\section{Statistical analysis}

All data were obtained from at least three independent experiments performed in triplicate. Statistical significance was determined using Student's $t$ test. $P$ values less than 0.05 were considered significant, and $P$ values less than 0.001 were considered highly significant.

\section{Results}

Prediction of three miRNA target sequences in the 3 '-UTR of human ADAMTS-4 mRNA

We used TargetScan 6.2 [22] to identify the miRNAs that target ADAMTS-4 mRNA. TargetScan 6.2 identified a sequence conserved in the 3'-UTR of ADAMTS-4 mRNA that was complementary to the miR-125b (Figure $1 \mathrm{~A}$ ), miR-125a and miR-4319 seed sequences (data not shown). We hypothesized that any of these miRNAs, if expressed in human chondrocytes may play an important role in regulating ADAMTS-4 expression.

\section{MiR-125b expression in normal and OA chondrocytes}

We determined the expression of miR-125b, miR-125a and miR-4319 using quantitative real-time PCR in normal and $\mathrm{OA}$ chondrocytes and cartilage tissues. In normal chondrocytes, miR-125b was strongly expressed while miR-125a and -4319 were barely detectable (Figure 1B). The expression of miR-125b was 49-fold higher than miR-125a and approximately two million-fold higher than miR-4319 (Figure 1B). Expression of miR-125b, but not miR-125a and -4319 , was found to be $54 \%$ lower in OA chondrocytes compared to normal chondrocytes (Figure 1C and data not shown). Compared to young normal cartilage tissue samples (mean age $=18.3$ years), the expression of miR-125b was $21 \%$ lower in normal cartilage tissue samples from older donors (mean age $=$ 50.7 years) and $85 \%$ lower in age-matched OA cartilage tissue samples (mean age $=57.7$ years) $($ Figure $1 \mathrm{C}$ ).

\section{IL-1 $\beta$ induces ADAMTS-4 expression and suppresses miR-125b expression}

We examined whether IL-1 $\beta$ affects the expression of ADAMTS-4, ADAMTS-5 and miR-125b. IL-1 $\beta$ 


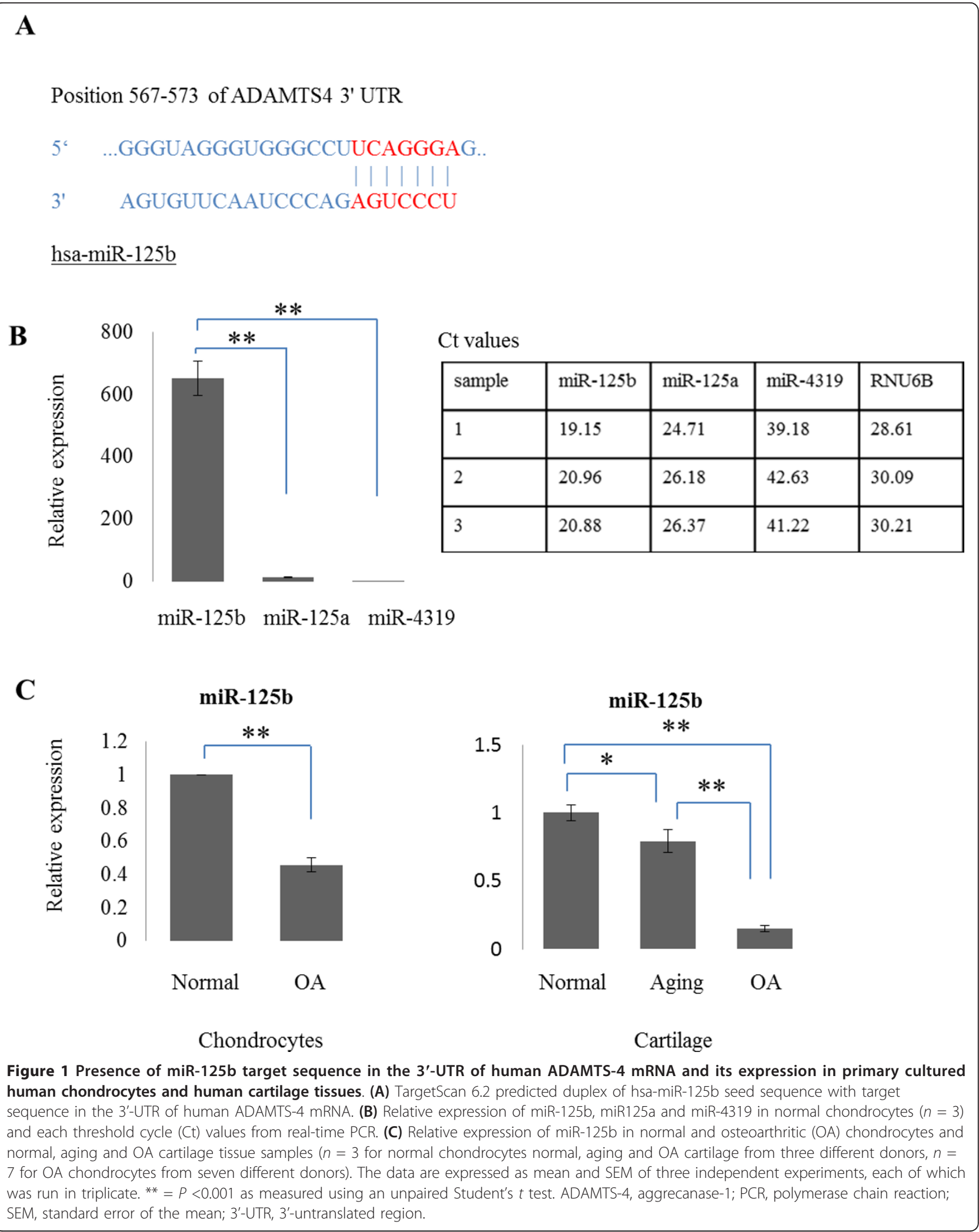




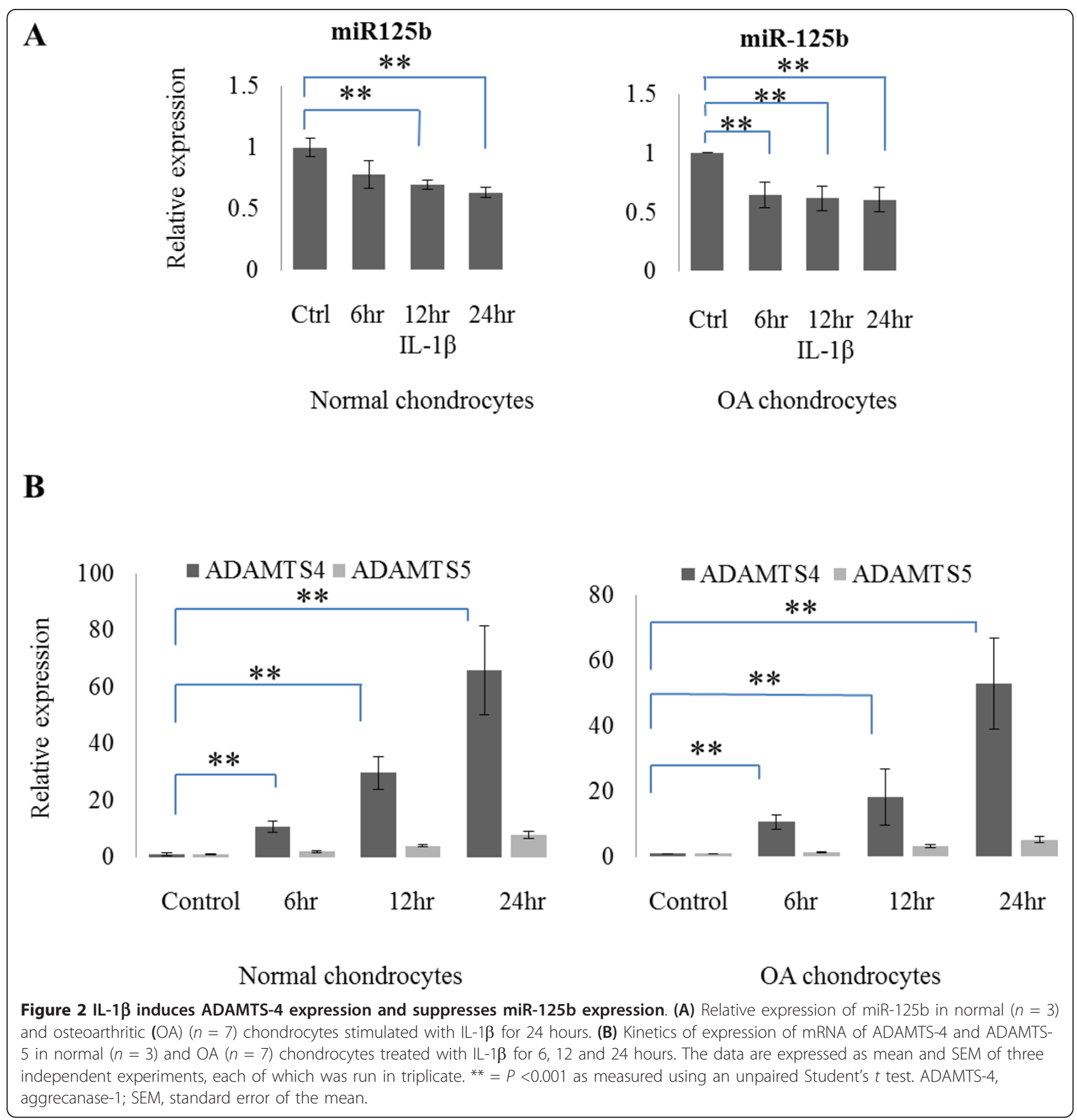

treatment suppressed miR-125b expression at 12 and 24 $\mathrm{h}$ in normal chondrocytes and at 6,12 and $24 \mathrm{~h}$ in OA chondrocytes $(P<0.001)$ (Figure $2 \mathrm{~A})$. IL-1 $\beta$ treatment induced ADAMTS-4 expression in a time-dependent manner in both normal and OA chondrocytes $(P$ $<0.001)$. ADAMTS-5 mRNA expression was also increased in a time-dependent manner, but the magnitude of induction was much lower than for ADAMTS-4 mRNA (Figure 2B).

\section{Regulation of ADAMTS4 mRNA expression by miR-125b} in human chondrocytes

To examine whether miR-125b regulates ADAMTS-4 in human normal and OA chondrocytes, we transfected the cells with pre-miR-125b or anti-miR-125b and treated with IL-1 $\beta$. Significant induction of miR-125b was observed in cells transfected with pre-miR-125b compared to negative control $(P<0.001)$, and suppression of miR$125 \mathrm{~b}$ was observed in cells transfected with anti-miR-125b 


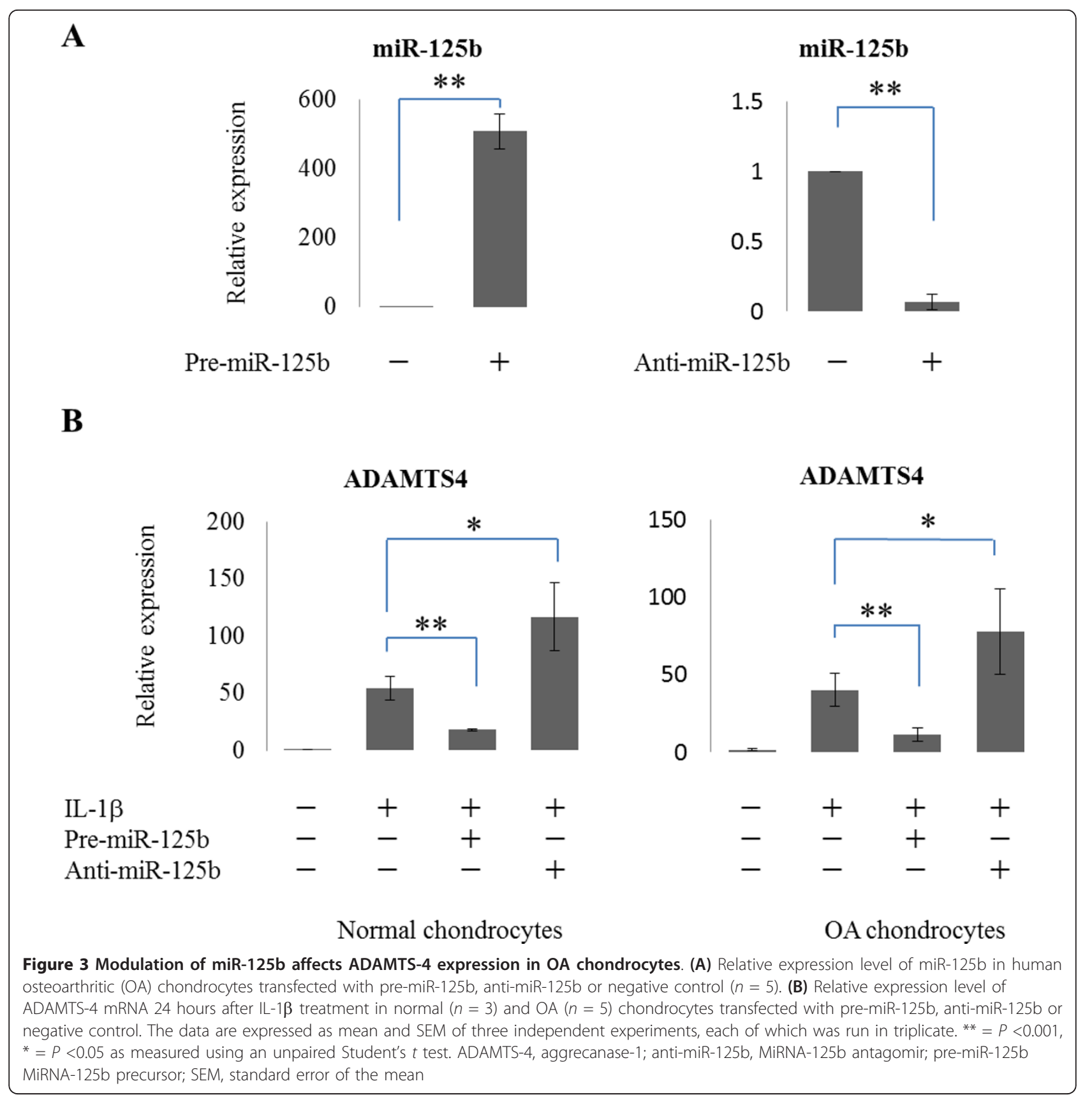

compared to negative control $(P<0.001)$ (Figure 3A). MiR$125 \mathrm{~b}$ decreased IL- $1 \beta$-induced ADAMTS- 4 mRNA ( $P$ $<0.001)$ (Figure 3B). On the other hand, anti-miR-125b increased IL-1 $\beta$-induced ADAMTS-4 mRNA $(P<0.05)$ (Figure 3B).

\section{Regulation of ADAMTS-4 protein by miR-125b in human chondrocytes}

To examine whether miR-125b also regulates ADAMTS4 protein levels, we performed western blotting on lysates from human OA chondrocytes transfected with miR-
$125 \mathrm{~b}$ with or without IL-1 $\beta$. In response to IL-1 $\beta$, ADAMTS-4 protein was increased (Figure 4A). In cells transfected with miR-125b, ADAMTS-4 protein was reduced by approximately $62 \%$ compared to cells transfected with control miRNA $(P<0.05)$ (Figure 4B and $C$ ).

\section{Functional validation of miR-125b using ADAMTS-4 3'-UTR luciferase reporter system}

To confirm that miR-125b regulates ADAMTS- 4 mRNA via directly binding to the predicted target sequence, we established a reporter system by combining luciferase 
A

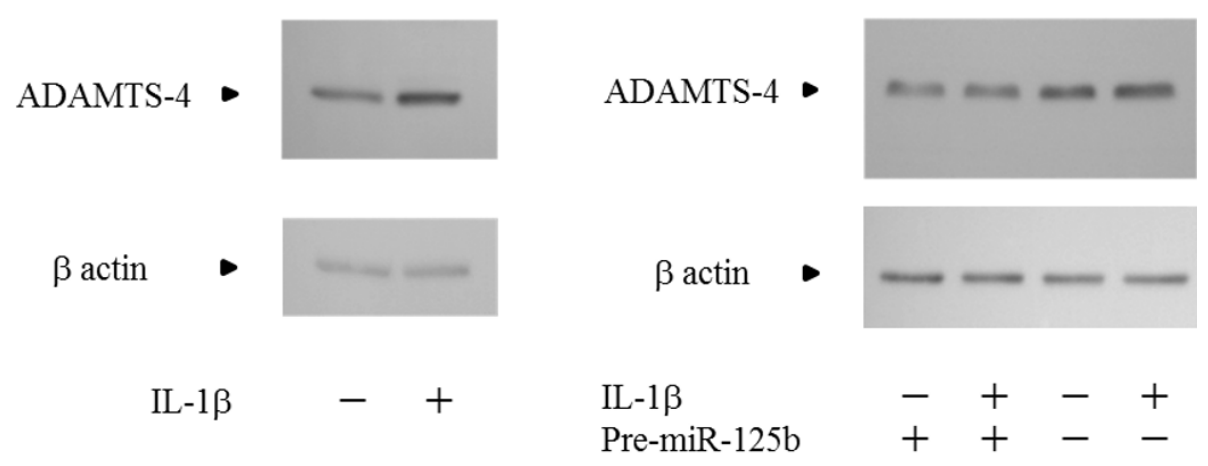

B

Pre-miR-125b ++-

C

ADAMTS-4/ק actin

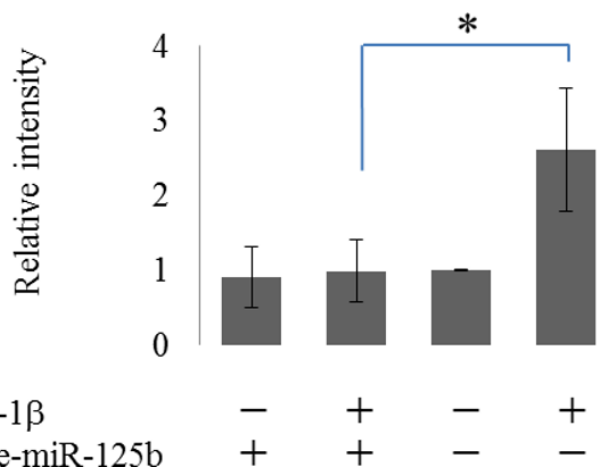

Figure 4 MiR-125b suppresses ADAMTS-4 protein in OA chondrocytes. (A) Increased production of ADAMTS-4 protein 24 hours after IL-1 $\beta$ treatment. (B) Osteoarthritic (OA) chondrocytes transfected with miR-125b or negative control incubated with or without IL-1 $\beta$ for 24 hours. (C) Bar graphs represent the average-fold increase \pm SD in pixel intensity of bands representing ADAMTS-4 normalized to $\beta$ actin, obtained from five separate western blot analyses for each cell condition. ${ }^{*}=P<0.05$ as measured using an unpaired Student's $t$ test. ADAMTS-4, aggrecanase-1; IL$1 \beta$, interleukin-1 beta; SD, standard deviation.

and the 3'-UTR of human ADAMTS-4 mRNA. As we identified two putative precursors of miR-125b as described in Materials and Methods, we validated both in this assay. Significant induction of miR-125b was confirmed in cells transfected with two putative precursors of miR-125b compared to negative control $(P<0.001)$ (Figure 5A). Compared to chondrocytes, 293T cells express very low levels of miR-125b (Figure 5B). We transiently co-transfected the ADAMTS-4 3'-UTR reporter construct or mutant reporter construct, and each pre-miR-125b or each mutant construct. The overexpression of miR-125b-1 or -2 markedly reduced luciferase activity compared to controls $(P<0.001)$ (Figure $5 \mathrm{C})$. We further confirmed these findings by using mutated forms of the 3'-UTR of human ADAMTS-4 mRNA and each miR-125b precursor. Transfection with the mutants abolished the suppression of luciferase activity (Figure $5 \mathrm{C}$ ). These results indicate that suppression is due to direct miR-125b binding to complementary seed sequence in ADAMTS-4 3'-UTR.

\section{Discussion}

In this study, we for the first time identified complementary sequence to miR-125b seed sequence in 3'UTR of human ADAMTS-4 mRNA. Our results show that miR-125b but not miR-125a is strongly expressed in normal human chondrocytes and cartilage tissues. Both miR-125a and miR-125b were previously reported to be highly expressed in mouse spinal cord and liver, but only miR-125b was detected in other tissues [23]. In humans, there are two miR-125b gene loci: miR-125b-1 on chromosome $11 \mathrm{q} 23$ and miR-125b-2 on chromosome $21 \mathrm{q} 21$, which are differentially expressed in a tissue-specific pattern. Although their pre-microRNAs have slightly different sequences, their mature sequences are identical [24]. 


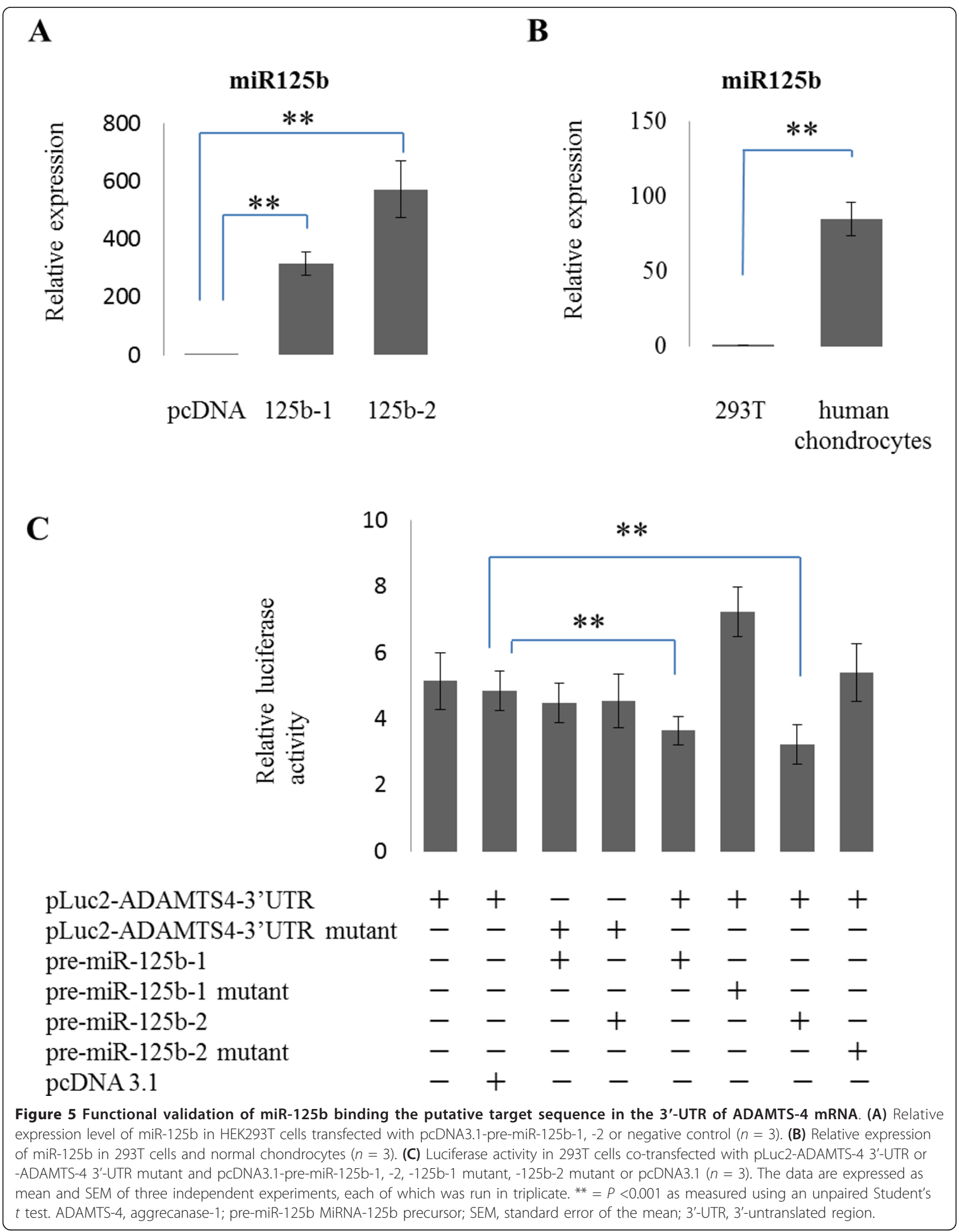


The present findings demonstrate that ADAMTS-4 is indeed regulated by $\mathrm{miR}-125 \mathrm{~b}$ via direct binding the predicted target sequence, resulting in suppression of ADAMTS-4 at both mRNA and protein level. The potential interaction between miR-125b and human ADAMTS4 mRNA was confirmed using luciferase reporter assay. In general, an 8 base-pair match is considered to represent a full-match seed sequence. For miR-125b and ADAMTS4 there is a 7 base-pair match, indicating partial complementarity. Computational and experimental evidence indicates that miRNA target sites with as little as 7 base pairs of complementarity to the miRNA 5 ' end are sufficient to confer regulation in vivo [25].

IL-1 $\beta$ is an important regulator of joint inflammation and cartilage degradation in $\mathrm{OA}$ and the present findings provide a link between IL- $1 \beta$ and miR-125b as chondrocyte treatment with IL-1 $\beta$ suppresses miR-125b expression, while inducing ADAMTS-4 expression. IL$1 \beta$ has also been reported to induce ADAMTS-4 mRNA levels with a dependency on NF- $\kappa$ B signaling [26]. In addition to this pathway, miR-125b might be another factor to regulate ADAMTS-4 mRNA expression in response to IL-1 $\beta$ signaling. Furthermore, we demonstrated that in primary human OA chondrocytes, overexpression of miR-125b leads to a significant reduction in IL-1 $\beta$ - induced ADAMTS-4 production. Collectively, the results indicate that $\mathrm{miR}-125 \mathrm{~b}$ directly regulates ADAMTS -4 in the context of activation by IL- $1 \beta$ as a key mediator of OA pathogenesis.

In this study, miR-125 suppressed ADAMTS-4 mRNA expression by $72 \%$ and protein production by $62 \%$ following IL-1 $\beta$ stimulation. In our previous study, miR140 suppressed ADAMTS-5 mRNA expression by similar degree with IL-1 $\beta$ stimulation [27]. It thus appears that the two main aggrecanases in cartilage are regulated by different miRNAs.

We also predicted target sequence of miR-125a and miR-4319 in 3'-UTR ADAMTS-4 mRNA. However, our results indicate that miR-125a and miR-4319 expression in chondrocytes is very low compared to miR-125b and there are no significant changes in OA chondrocytes (data not shown). Thus, miR-125b, but not miR-125a and miR-4319, may play a role in OA development. The expression of miR-125b was reduced with a dependency on age, but the expression of miR-125b more significantly decreased in age-matched OA cartilage tissues compared to young normal cartilage tissues. The expression of miR$125 \mathrm{~b}$ seems to be influenced by age-related factors, but more strongly by mechanisms related to OA.

The protective role of miR-125b in cartilage may extend beyond the suppression of ADAMTS-4. In other cell types miR-125b also suppressed MMP-13 and vascular endothelial cadherin [28,29]. MMP-13 is an important collagenase in articular cartilage [30], and vascular endothelial cadherin regulates angiogenesis, which is increased in OA-affected joints [31].

\section{Conclusions}

This is the first report identifying a miRNA, miR-125b, as a posttranscriptional regulator of ADAMTS-4 in human chondrocytes. We have demonstrated the two precursors of miR-125b in human genome suppress the expression of ADAMTS-4 mRNA and protein. We suggest that to controlling the expression of miR-125b has potential as a novel approach for prevention and treatment of OA.

\section{Abbreviations}

ADAMTS: a disintegrin and metalloproteinase with thrombospondin motifs; ADAMTS-4: aggrecanase-1; ADAMTS-5: aggrecanase-2; anti-miR-125b: MiRNA125b antagomir; cDNA: complementary DNA; CIP: calf intestinal phosphatase; DMEM: Dulbecco's modified Eagle's medium; dNTP: deoxynucleoside triphosphate; ECM: extracellular matrix; FBS: fetal bovine serum; GAPDH: glyceraldehyde 3-phosphate dehydrogenase; IL-1 $\beta$ : interleukin-1 beta; MiRNA: microRNA; MMP: matrix metalloproteinase; mRNA: messenger RNA; OA: osteoarthritis; pre-miR-125b: MiRNA-125b precursor; RNU6B: U6 small nuclear RNA; TNF-a: tumor necrosis factor-alpha; 3'-UTRs: 3'untranslated regions.

\section{Authors' contributions}

The study was designed by TS. TM and TY contributed to the acquisition of the data, analysis and interpretation, and drafting of the manuscript. TS, MKL, $\mathrm{HA}$ and $\mathrm{NI}$ contributed to the conception and interpretation of the article and the obtaining of funds for this study. $\mathrm{HN}, \mathrm{HH}, \mathrm{TH}, \mathrm{MN}, \mathrm{YO}, \mathrm{SI}$ contributed to the samples collection, acquisition of the data, analysis and interpretation of the manuscript. All authors contributed to the revising of the manuscript and approved the final manuscript.

\section{Competing interests}

The authors have declared that they have no competing interests.

\section{Acknowledgements}

Funding was supported by the Japan Society for the Promotion of Science KAKENHI 22659271 (Grant-in-Aid for Challenging Exploratory Research), the MEISEIKAI fund, JSPS (G2216, TM) and NIH grants AR-50631 (HA) and AG007996 (ML). HA was supported by a grant from Health and Labor Sciences Research Grants, Grants-in Aid for Scientific Research (MEXT) and JST, CREST, NIH AR050631, AR056120. TM and HA was supported by a grant from The Grant of National Center for Child Health and Development (20A3), Strategic Young Researcher Overseas Visits Program for Accelerating Brain Circulation (JSPS).

\section{Author details}

'Department of Orthopaedic Surgery, Nagoya University Graduate School of Medicine, 65 Tsurumai-cho, Showa-ku, Nagoya 466-8550, Japan. ${ }^{2}$ Department of Molecular and Experimental Medicine, The Scripps Research Institute, 10550 North Torrey Pines Road, La Jolla, CA 92037, USA. ${ }^{3}$ Department of System Biomedicine, Tokyo Medical and Dental University, 1-5-45 Tsushima, Bunkyo-ku, Tokyo 113-8510, Japan. ${ }^{4}$ Department of System Biomedicine, National Research Institute for Child Health and Development, 2-10-1 Okura, Setagaya-ku, Tokyo 157-8535, Japan.

Received: 16 October 2012 Revised: 10 January 2013

Accepted: 7 February 2013 Published: 13 February 2013

\section{References}

1. Dahlberg $L$, Billinghurst RC, Manner $P$, Nelson F, Webb G, lonescu M, Reiner A, Tanzer M, Zukor D, Chen J, van Wart HE, Poole AR: Selective enhancement of collagenase-mediated cleavage of resident type II collagen in cultured osteoarthritic cartilage and arrest with a synthetic 
inhibitor that spares collagenase 1 (matrix metalloproteinase 1). Arthritis Rheum 2000, 43:673-682.

2. Tortorella MD, Malfait AM, Deccico C, Arner E: The role of ADAM-TS4 (aggrecanase-1) and ADAM-TS5 (aggrecanase-2) in a model of cartilage degradation. Osteoarthr Cartil 2001, 9:539-552.

3. Pratta MA, Yao W, Decicco C, Tortorella MD, Liu RQ, Copeland RA, Magolda R, Newton RC, Trzaskos JM, Arner EC: Aggrecan protects cartilage collagen from proteolytic cleavage. J Biol Chem 2003, 278:45539-45545.

4. Porter S, Clark IM, Kevorkian L, Edwards DR: The ADAMTS metalloproteinases. Biochem J 2005, 386:15-27.

5. Sandy JD: A contentious issue finds some clarity: on the independent and complementary roles of aggrecanase activity and MMP activity in human joint aggrecanolysis. Osteoarthr Cartil 2006, 14:95-100.

6. Song RH, Tortorella MD, Malfait AM, Alston JT, Yang Z, Arner EC, Griggs DW: Aggrecan degradation in human articular cartilage explants is mediated by both ADAMTS-4 and ADAMTS-5. Arthritis Rheum 2007, 56:575-585.

7. Naito S, Shiomi T, Okada A, Kimura T, Chijiiwa M, Fujita Y, Yatabe T, Komiya K, Enomoto H, Fujikawa K, Okada Y: Expression of ADAMTS4 (aggrecanase-1) in human osteoarthritic cartilage. Pathol Int 2007, 57:703-711.

8. Zhou PH, Liu SQ, Peng H: The effect of hyaluronic acid on IL-1 betainduced chondrocyte apoptosis in a rat model of osteoarthritis. J Orthop Res 2008, 26:1643-1648.

9. Goldring MB: Osteoarthritis and cartilage: the role of cytokines. Curr Rheumatol Rep 2000, 2:459-465.

10. Lopez-Armada MJ, Carames B, Lires-Dean M, Cillero-Pastor B, RuizRomero C, Galdo F, Blanco FJ: Cytokines, tumor necrosis factor-alpha and interleukin-1 beta, differentially regulate apoptosis in osteoarthritis cultured human chondrocytes. Osteoarthrs Cartil 2006, 14:660-669.

11. Zwerina J, Redlich K, Polzer K, Joosten L, Kronke G, Distler J, Hess A, Pundt N, Pap T, Hoffmann O, Gasser J, Scheinecker C, Smolen JS, Berg W, Schett G: TNF-induced structural joint damage is mediated by IL-1. Proc Natl Acad Sci USA 2007, 104:11742-11747.

12. Goldring MB, Birkhead J, Sandell $\sqcup$, Kimura T, Krane SM: Interleukin 1 suppresses expression of cartilage-specific types II and IX collagens and increases types I and III collagens in human chondrocytes. J Clin Invest 1988, 82:2026-2037.

13. Lefebvre V, Peeters-Joris C, Vaes G: Modulation by interleukin 1 and tumor necrosis factor alpha of production of collagenase, tissue inhibitor of metalloproteinases and collagen types in differentiated and dedifferentiated articular chondrocytes. Biochimica et biophysica acta 1990, 1052:366-378.

14. Lagos-Quintana M, Rauhut R, Lendeckel W, Tuschl T: Identification of novel genes coding for small expressed RNAs. Science 2001, 294:853-858.

15. Bartel DP: MicroRNAs: genomics, biogenesis, mechanism, and function. Cell 2004, 116:281-297.

16. Lee RC, Ambros V: An extensive class of small RNAs in Caenorhabditis elegans. Science 2001, 294:862-864.

17. Lau NC, Lim LP, Weinstein EG, Bartel DP: An abundant class of tiny RNAs with probable regulatory roles in Caenorhabditis elegans. Science 2001, 294:858-862.

18. Kim VN: MicroRNA biogenesis: coordinated cropping and dicing. Nat Rev Mol Cell Biol 2005, 6:376-385.

19. Miyaki S, Sato T, Inoue A, Otsuki S, Ito Y, Yokoyama S, Kato Y, Takemoto F, Nakasa T, Yamashita S, Takada S, Lotz MK, Ueno-Kudo H, Asahara H: MicroRNA-140 plays dual roles in both cartilage development and homeostasis. Genes Dev 2010, 24:1173-1185.

20. Akhtar N, Rasheed Z, Ramamurthy S, Anbazhagan AN, Voss FR, Haqqi TM: MicroRNA-27b regulates the expression of matrix metalloproteinase 13 in human osteoarthritis chondrocytes. Arthritis Rheum 2010, 62:1361-1371.

21. UCSC genome browser. [http://genome.ucsc.edu].

22. TargetScan 6.2. [http://www.targetscan.org].

23. Tang $X$, Gal J, Zhuang $X$, Wang W, Zhu H, Tang G: A simple array platform for microRNA analysis and its application in mouse tissues. RNA 2007 13:1803-1822.

24. Puissegur MP, Eichner R, Quelen C, Coyaud E, Mari B, Lebrigand K Broccardo C, Nguyen-Khac F, Bousquet M, Brousset P: B-cell regulator of immunoglobulin heavy chain transcription (Bright)/ARID3a is a direct target of the oncomir microRNA-125b in progenitor B-cells. Leukemia 2012, 26:2224-32.
25. Brennecke J, Stark A, Russell RB, Cohen SM: Principles of microRNA-target recognition. PLOS Biol 2005, 3:e85.

26. Chockalingam PS, Varadarajan U, Sheldon R, Fortier E, LaVallie ER, Morris EA, Yaworsky PJ, Majumdar MK: Involvement of protein kinase Czeta in interleukin-1 beta induction of ADAMTS-4 and type 2 nitric oxide synthase via NF-kappaB signaling in primary human osteoarthritic chondrocytes. Arthritis Rheum 2007, 56:4074-4083.

27. Miyaki S, Nakasa T, Otsuki S, Grogan SP, Higashiyama R, Inoue A, Kato Y, Sato T, Lotz MK, Asahara H: MicroRNA-140 is expressed in differentiated human articular chondrocytes and modulates interleukin-1 responses. Arthritis Rheum 2009, 60:2723-2730.

28. Xu N, Zhang L, Meisgen F, Harada M, Heilborn J, Homey B, Grander D, Stahle M, Sonkoly E, Pivarcsi A: MicroRNA-125b down-regulates matrix metallopeptidase 13 and inhibits cutaneous squamous cell carcinoma cell proliferation, migration, and invasion. J Biol Chem 2012, 287:29899-29908.

29. Muramatsu F, Kidoya H, Naito H, Sakimoto S, Takakura N: microRNA-125b inhibits tube formation of blood vessels through translational suppression of VE-cadherin. Oncogene 2012, 32:414-21.

30. Little CB, Barai A, Burkhardt D, Smith SM, Fosang AJ, Werb Z, Shah M, Thompson EW: Matrix metalloproteinase 13-deficient mice are resistant to osteoarthritic cartilage erosion but not chondrocyte hypertrophy or osteophyte development. Arthritis Rheum 2009, 60:3723-3733.

31. Mapp PI, Walsh DA: Mechanisms and targets of angiogenesis and nerve growth in osteoarthritis. Nature reviews Rheumatology 2012, 8:390-398.

doi:10.1186/ar4164

Cite this article as: Matsukawa et al: MicroRNA-125b regulates the expression of aggrecanase-1 (ADAMTS-4) in human osteoarthritic chondrocytes. Arthritis Research \& Therapy 2013 15:R28.

\section{Submit your next manuscript to BioMed Central and take full advantage of:}

- Convenient online submission

- Thorough peer review

- No space constraints or color figure charges

- Immediate publication on acceptance

- Inclusion in PubMed, CAS, Scopus and Google Scholar

- Research which is freely available for redistribution 